\title{
A Quantitative Bohman-Korovkin Theorem and its Sharpness for Riemann Integrable Functions
}

\author{
H. Mevissen, R. J. Nessel and E. van Wickeren
}

Ausgehend von einer Arbeit von Pólya (1933) über die Konvergenz von Quadraturverfahren haben wir kürzlich einen Folgenkonvergenzbegriff im Raum der Riemann-integrierbaren Funktionen eingeführt, unter dem sich dieser klassische Raum als Vervollständigung der ste: tigen Funktionen darstellt. In der sich dann anschließenden Diskussion möglicher Auswirkungen auf dié Approximationstheorie.wurde unter anderem der Satz'von Bohman-Korovkin (mit. und ohne Ordnung) von stetigen auf Riemann-integrierbare Funktionen übertragen. Die dabei erziclten Schranken wurden über den ersten $\tau$-Modul ausgedrückt. Ein Ziel dieser Arbeit ist es, entsprechende Abschätzungen gegen den zweiten $\tau$-Modul herzuleiten. Die Schärfe dieser Resultate wird dann mittels eines quantitativen Beschränktheitsprinzips gezeigt. Schließlich werden die allgemeinen Ergebnisse im Zusammenhang mit Bernstein-Polynomen und linearen, interpolierenden Splines getestet.

Исходя от одной работы Полиа (1933) о сходимости методов квадратур мы ввели недавно понлтие сходимости последовательностей в пространстве интегрируемых по Риману функций, при котором әто классическое пространство оказалось дополнением непрерывных функций. В ходе последующе дискуссии о возмонных последствиях на теорию аппроксимации переносилась, между прочим, теорема Бомана-Коровкина (с порядком и без него) „с непрерывных на 'интегрируемые по Риману функций. Достигнутые при этом границы погрешности выражались через первып т-модуль. Одна из целей этой работы - выводить соответствующие оценки отиосительно второго $\tau$-модуля. Потом посредством количественного принципа ограниченности показывается что эти резуль. таты являются точными. Наконец, общие результаты проверяются в свяси с полиномами Бернштейна и линейнмии интерполирующими сплайнайи.

Inspired by work of Polya (1933) on the convergence of quadrature formulas, we previously introduced a concept of sequential convergence in the space of Riemann integrable functions under which this classical space is in fact the completion of continuous functions. Discussing its consequences to approximation theory, among others we already extended the (qualitative as well as quantitative) Bohman-Korovkin theorem from continuous to Riemann integrable functions. The error bounds obtained were given in terms of the first $\tau$-modul. One purpose of this paper is to derive corresponding results, involving the second $\tau$-modul. As a consequence of our previous quantitative uniform boundedness principle it is then shown that these estimates are indeed sharp. The gencral results obtained are illustrated in connection with Bernstein polynomials and linear interpolating splines.

\section{Introduction}

Let $B=B[a, b]$ and $R=R[a, b]$ be the spaces of functions, everywhere defined on the compact interval $[a, b]$ of the real axis $\mathbf{R}$ which are bounded and Riemann integrable, respectively. Though $B$ and $R$ are Banach spaces under the norm $\|f\|_{B}$ $:=\sup \{|f(u)|: u \in[a, b]\}$, uniform convergence is not appropriate to approximate in $R$ since then suitable dense subsets are not available. This is a voided by the following concept of sequential convergence: A sequence $\left\{f_{n}\right\}^{-} \subset B$ is called (Riemann) $R$-con- 
vergent to $f \in B$ if for $n \rightarrow \infty$

$$
\left\|f_{n}\right\|_{B}=O(1), \quad \bar{\int} \sup _{k \geqq n}\left|f_{k}-f\right|=o(1)
$$

with upper Riemann integral $\bar{\int} f:=\int_{a}^{b} f(u) d u$. It turns out that $B$ as well as $R$ are still (sequentially) $R$-complete. From the point of view of approximation, the most important feature then is that the $R$-closure of standard classes of smooth functions (e.g., polynomials) yields $R$. In fact, Banach-Steinhaus theorems, can be established in this framework, giving necessary and sufficient conditions for the $R$-convergence of operators. See [6] for the details.

Turning to positive operators, it was shown in [12] that the well-known BohmanKorovkin theorem remains valid in the present context. Indeed, a sequence $\left\{T_{n}\right\}$ of positive linear operators of $R$ into itself is $R$-convergent on $R$ if and only if the sequence is $R$-convergent for the three test functions $p_{i}(u):=u^{i}, i=0,1,2$.

Concerning quantitative extensions, an appropriate measure of smoothness is given by the $k$-th $\tau$-modul $(f \in B, \delta>0, k \in \mathbf{N})$

$$
\begin{aligned}
& \tau_{k}(f, \delta)=\int_{a}^{b} \omega_{k}(f, x, \delta) d x, \quad \omega_{k}(j, x, \delta)=\sup \left\{\left|\Lambda_{h}{ }^{k} f(y)\right|: y \pm \frac{k^{\prime}}{2} h \in U_{\delta}(x)\right\} \\
& \dot{\Delta}_{h}{ }^{k} f(y)=\sum_{j=0}^{k}(-1)^{k-j}\left(\begin{array}{l}
k \\
j
\end{array}\right) f\left(y+\left(j-\frac{k}{2}\right) h\right) \\
& \left.U_{\delta}(x)=[x-\delta, x+\delta)\right] \cap[a, b],
\end{aligned}
$$

employed by the Bulgarian school of approximation during the last decade (see [15], there for functions $f$, measurable and bounded). Indeed, $\tau_{1}(f, \delta)=c(1)$ for $\delta \rightarrow 0+$ if and only' if $f \in R$. The following error bound was established in [12].

Theorem 1: Let $\left\{T_{n}\right\}$ be a sequence of positive linear operators, mapping $R$ into itself such that $T_{n} p_{0}=p_{0}$. Setting

$$
\mu_{n}:=\sup _{k \geq n}\left\|T_{k}\left((u-x)^{2} ; x\right)\right\|_{B},
$$

for each $f \in R$ there hold true the estimates

$$
\begin{aligned}
& \left\|T_{n} f\right\|_{B} \leqq\|f\|_{B} ; \\
& \int \sup _{k \geqq n}\left\{T_{k} f-f \mid \leqq 5 \tau_{1}\left(f, \mu_{n}^{1 / 2}\right)\right.
\end{aligned}
$$

Obviously, this result is again completely parallel to the classical one, concerned with continuous functions and uniform convergence (see $[3$, p. 28] and the literature cited there): In Section 2 we improve (3) to all estimate in volving the second $\tau$-modul, again quite parallel to the procedure in the continuous case (see $[8 ; 9]$ and the literature cited there). This, however, can only be done for a special class of positive operators, characterized by condition (9) on the fourth moment (see the comments, given at the end of Section 2). Applying our previous quantitative extensions of the uniform boundedness principle, it is then shown in Section 3 that the preceding estimates are indeed sharp. Finally, in Section 4 we test the general results in connection with Bernstein polynomials and linear interpolating. splines (see also the hints to the literature, given there). 


\section{The Bohman-Korovkin theorem}

To derive the estimate mentioned we proceed along the standard interpolation argument. To this end, define a norm for $f \in R$ and $\delta>0$ via

$$
\|f\|_{\delta}=\int_{a}^{b} M(f, x, \delta) d x, \quad M(f, x, \delta)=\sup _{\nu \in U_{\delta}(x)}|f(y)|,
$$

and let $R^{2}=R^{2}[a, b]$ be the space of functions $g$, twice differentiable on $[a, b] \cdot$ with $g^{\prime \prime}, \in R$, endowed with the seminorm $|g|_{2}:=\int_{a}^{0}\left|g^{\prime \prime}(\dot{x})\right| d x$. The following lemma is well-known and may be found in $[1 ; 2 ; 13]$. Let us include a proof for the sake of completeness (and simplicity).

Lemma 2: For $f \in R, 0<\delta<b-a$ there exists $g_{\delta} \in \dot{R}^{2}$ such that

$$
\left\|f-g_{\delta}^{\prime}\right\|_{\delta} \leqq 10 \tau_{2}(f, \delta), \quad . \quad\left|g_{\delta}\right|_{2} \leqq 20 \delta^{-2} \tau_{2}(f, \delta) .
$$

Proof: First of all note that -

$$
\|f\|_{\delta} \leqq 2\|f\|_{\delta / 2} \quad(f \in R) .
$$

Indeed; one has

$$
\dot{M}(f, x, \delta) \leqq \begin{cases}M(f, x-\delta / 2, \delta / 2)+M(f, x+\delta / 2, \delta / 2), & a+\delta / 2 \leqq x \leqq b-\delta / 2, \\ M(f, x+\delta / 2, \delta / 2)+M(f, x, \delta / 2), & a \leqq x \leqq a+\delta / 2, \\ M(f, x-\delta / 2, \delta / 2)+M(f, x, \delta / 2), & b-\delta / 2 \leqq x \leqq b,\end{cases}
$$

which yields

$$
\|f\|_{\delta} \leqq\left(\int_{a}^{b-\delta}+\int_{a+\delta}^{b}+\int_{a+\delta / 2}^{a+\delta}+\int_{a}^{a+\delta / 2}+\int_{\delta-\delta}^{b-\delta / 2}+\frac{b}{b-\delta / 2}\right) M(f, x, \delta / 2) d x=2\|f\|_{\delta / 2} .
$$

Now define Steklov means for $f \in R$ via

$$
g_{\delta}(x)=4 \delta^{-2} \int_{-\delta / 4}^{\delta / 4} \bar{f}(x+s+t) d s d t=4 \delta^{-2} \int_{-\delta / 4}^{\delta / 4} \bar{f}(x-s-t) d s d t
$$

where $f$ is given as the extension (cf. $[16, p, 121]$ )

$$
\bar{f}(x)= \begin{cases}f(x), & a \leqq x \leqq b, \\ -f(2 a-x)+2 f(a), & 2 a-b \leqq x \leqq a, \\ -f(2 b-x)+2 f(b), & \dot{b} \leqq x \leqq: 2 b-a .\end{cases}
$$

Since for $y \pm h \in[x-\delta, x+\delta]$ the difference $\Delta_{h}^{2} \bar{f}(y)$ only differs from $\Delta_{h}^{2} f(y)$ if, e.g., $y-h<a \leqq y \leqq y+h$, and since in this special case

$$
\Delta_{h}^{2} \bar{f}(y)=\Delta_{y-a}^{2} f(a+\ddot{h})-2 \Delta_{y-a-h / 2}^{2} f(a+h / 2)+2 \Delta_{h / 2}^{2} f(a+h / 2),
$$

it follows that for $x \in[a, b]$

$$
\bar{\omega}_{2}(\bar{f}, x, \delta) \leqq 5 \omega_{2}(f, x, \delta), \quad \bar{\omega}_{2}(\bar{f}, x, \delta):=\sup _{y, y \pm h \in\lfloor x-\delta, x+\delta \mid}\left|\Delta_{h}^{2} \bar{f}(y)\right| \cdot
$$

In view of $(6)$ one obtains for $y \in U_{\delta / 2}(x)$

$$
\left|g_{\delta}(y)-f(y)\right|=2 \delta^{-2}\left|\int_{-\delta / 4}^{\delta / 4} A_{s+l}^{2} \bar{f}(y) d s d t\right| \leqq \bar{\omega}_{2}(\bar{f}, x, \delta)
$$


which delivers (cf. (5), (7)) $M\left(g_{\delta}-f, x, \delta / 2\right) \leqq 5 \omega_{2}(f, x, \delta)$, $\left\|f^{\prime}-g_{\delta}\right\|_{\delta} \leqq 10 \tau_{2}(f, \delta)$. Moreover, $g_{\delta} \in R^{2}$ with $\left|g_{\delta}^{\prime \prime}(x)\right|=4 \delta^{-2}\left|\mathcal{L}_{\delta}^{2} \bar{f}(x)\right| \leqq 4 \delta^{-2} \bar{\omega}_{2}(\bar{f}, x, \delta)$, which implies (4) in view of (7)

We are now in the position to prove the following quantitative Bohman-Korovkin theorem.

Theorem 3: Let $\left\{T_{n}\right\}$ be a sequence of positive linear operators, mapping $R$-into itself such that (cf. (1))

$$
\begin{aligned}
& T_{n} p_{i}=p_{i} \quad(i=0,1), \\
& \left\|T_{n}\left((u-x)^{4} ; x\right)\right\|_{B} \leqq K \mu_{n}{ }^{2}
\end{aligned}
$$

for some constant $K>0$. Then there holds true the estimate

$$
\bar{\int} \sup _{k \geqq n}\left|T_{k} f-f\right| \leqq C \tau_{2}\left(f, \mu_{n}^{1 / 2}\right) \quad(f \in R)
$$

Proof: In view of Lemma 2 and

$$
\bar{\int} \sup _{k \geq n}\left|T_{k} f-f\right| \leqq \bar{\int} \sup _{k \geq n}\left|T_{k}\left(f-g_{\delta}\right)-\left(f-g_{\delta}\right)\right|+\bar{\int} \sup _{k \geq n}\left|T_{k} g_{\delta}-g_{\delta}\right|
$$

it is sufficient to show that $\left(\delta=\mu_{n}^{1 / 2}\right)$

$$
\begin{aligned}
& \bar{\int} \sup _{k \geq n}\left|T_{k}\left(f-g_{\delta}\right)-\left(f-g_{\delta}\right)\right| \leqq 10\left\|f-g_{\delta}\right\|_{\mu_{n}} \overline{3 / 2}, \\
& \bar{\int} \sup _{k \geq n}\left|T_{k} g_{\delta}-g_{\delta}\right| \leqq C \mu_{n}\left|g_{\delta}\right|_{2} .
\end{aligned}
$$

Obviously, (11) is a consequence of Theorem 1 since $\tau_{1}(f, \delta) \leqq 2\|f\|_{\delta}$. To establish (12) consider the expansion $\left(g=g_{j}, u, x \in[a, b]\right)$

$$
g(u)=g(x)+(u-x) g^{\prime}(x)+\int_{x}^{u}(u-t) g^{\prime \prime}(t) d t .
$$

Concerning the remainder one has

$$
\begin{aligned}
I & :=\left|\int_{x}^{u}(u-t) g^{\prime \prime}(t) d t\right| \leqq|u-x|\left|\int_{x}^{u}\right| g^{\prime \prime}(t)|d t| \\
& \leqq\left(\delta+\frac{(u-x)^{2}}{\delta}\right) \int_{|t-x|<\delta}\left|g^{\prime \prime}(t)\right| d t+(u-x)^{4} \int_{|t-x| \geqq \delta}\left|g^{\prime \prime}(t)\right| \frac{d t}{|t-x|^{3}}=: I_{1}+I_{2}
\end{aligned}
$$

It follows that for $k \geq n$ (cf. (8))

and therefore

$$
T_{k}\left(\dot{I}_{1} ; x\right)=\left(\delta+\frac{T_{k}\left((u-x)^{\dot{2}} ; x\right)}{\delta}\right) \int_{|t-x|<\delta}\left|g^{\prime \prime}(t)\right| d t \leqq\left(\delta+\frac{\mu_{n}}{\delta}\right) \int_{|t-x|<\delta} g^{\prime \prime}(t) \mid d t
$$

$$
\int \sup _{k \geqq n} T_{k} I_{1} \leqq\left(\delta+\frac{\mu_{n}}{\delta}\right) \iint_{|\ell-x|<\delta}\left|g^{\prime \prime}(t)\right| d t d x=\left(\delta+\frac{\mu_{n}}{\delta}\right) 2 \delta|g|_{2} .
$$


Furthermore, in view of (9) one obtains that for $k \geqq n$

$$
\begin{aligned}
& T_{k}\left(I_{2} ; x\right) \leqq K \mu_{n}^{2} \int_{|t-x| \geqq \delta}\left|g^{\prime \prime}(t)\right| \frac{d t}{|t-x|^{3}}, \\
& \int_{k} \sup _{k \geqq n} T_{k} I_{2} \leqq K \mu_{n}^{2} \int_{|t-x| \geqq \delta} \int_{\left|g^{\prime \prime}(t)\right| \frac{d t}{|t-x|^{3}} d x \leqq K \mu_{n}^{2} \delta^{-2}|g|_{2} .}^{;}
\end{aligned}
$$

Together with (8), (13) this delivers

$$
\bar{\int} \sup _{k \geq n}\left|T_{k} g-g\right| \leqq\left(\delta+\frac{\mu_{n}}{\delta}\right) 2 \delta|g|_{2}+K \mu_{n}^{2} \delta^{-2} \cdot|g|_{2},
$$

- which establishes (12) by setting $\delta=\mu_{n}^{1 / 2}$.

In view of $(10)$ condition (8) is somehow natural (cf. $[14$, p. 18]) since then

$$
\bar{\int} \sup _{k \geq n}\left|T_{k} p_{i}-p_{i}\right| \leqq C \tau_{2}\left(p_{i}, \mu_{n}^{1 / 2}\right)=0 .
$$

Thus the relevant assumption is condition (9), well-known in the literature in connection with a treatment of related problems such as saturation or in verse theorems (cf. $\left[3\right.$, p. 131, 264], [4]). Note that if $T_{n} p_{0}=p_{0}$, then conversely $T_{n}\left((u-x)^{2} ; x\right)^{2}$ $\leqq T_{n}\left((u-x)^{4} ; x\right)$ by the Cauchy-Schwarz inequality. On the other hand, without condition (9) the best result; known so far, is

$$
\int\left|T_{n} f-f\right| \leqq C \tau_{2}\left(f,\left(v_{n}\left|\log \nu_{n}\right|\right)^{1 / 2}\right), \quad v_{n}:=\left\|T_{n}\left((u-x)^{2} ; x\right)\right\|_{B},
$$

given in $[14$, p. 45].

\section{The sharpness}

To derive the sharpness of Theorem 3 , a quarititative extension of the uniform boundedness principle will be applied. To this end, let $\omega(\delta)$ be an abstract modulus of continuity, i.e., $\omega(\delta)$ is a positive, continuous, subadditive, and increasing function of $\delta>0$ satisfying

$$
\omega(\delta) \doteq c(1), \quad \delta=\cdot(\omega(\delta)) \quad(\delta \rightarrow 0+) .
$$

Furthermore, let $X$ be a Banach space with norm $\|\cdot\|_{X}$ and $X^{*}$ be the space of nonnegative, sublinear, bounded functionals $T$ on $X$, i.e., for $f, g \in X$ and scalars $\alpha$

$$
\begin{aligned}
& 0 \leqq T(f+g) \leqq T f+T g, \quad T(\dot{\alpha} f)=|\alpha| T f, \\
& \sup \left\{T f: f \in X,\|f\|_{x} \leqq 1\right\}<\infty .
\end{aligned}
$$

The following resonance principle holds true (cf. [7] and the literature cited there).

Theorem 4: Let $\left\{\varphi_{n}\right\}$ be a decreasing nullsequence, let $\sigma(\delta)>0$ and $\omega(\delta)$ be subject to (14). Suppose that for $U_{\mathrm{d}}, R_{n} \in X^{*}$ there are elements $h_{n} \in \cdot X$ satisfying

$$
\begin{array}{ll}
\left\|h_{n}\right\|_{X}=O(1), \\
U_{\delta} h_{n} \leqq M \min \left\{1, \sigma(\delta) / \varphi_{n}\right\} . \\
R_{n} h_{n} \neq \circ(1) . \quad(\delta .>0, n \in \mathbf{N}),
\end{array}
$$


Then there exists a counterexample $f_{\omega} \in X$ with

$$
U_{\delta} f_{\omega}=O(\omega(\sigma(\delta))), \quad R_{n} f_{\omega} \neq o\left(\omega\left(\varphi_{n}\right)\right) .
$$

In the present context $X$ will be the Banach space $R$ with norm $\|\cdot\|_{B}, U_{\delta} f=\tau_{2}(f, \delta)$ with $\varphi_{n}=\mu_{n}$ and $\sigma(\delta)=\delta^{2}$, and $R_{n} f=\bar{\int} \sup _{k \geqq n}\left|T_{k} f-f\right|$. The sharpness of the estimate $(10)$ is then established by

Theorem 5: Let $\left\{T_{n}\right\}$ be a sequence of positive linear operators of $R$ into itself, satisfying (8), (9) with $\mu_{n}=c(1)$. (cf. (1)). Moreover, for each $n \in \mathbf{N}$ let $I_{n}$ be the union of $j_{n}$ disjoint subintervals $I_{j n}:=\left(a_{j n}, b_{j n}\right)$ of $[a, b]$ such that ,

$$
\begin{aligned}
& 0<c_{1} \leqq j_{n} \mu_{n}^{1 / 2} \leqq c_{2} ; \quad b_{i n}-a_{j n} \geqq c_{3} \bar{\mu}_{n}^{1 / 2}>0, \\
& 0<c_{4} \mu_{n} \leqq T_{n}\left((u-x)^{2} ; x\right) \quad\left(x \in I_{n}\right) .
\end{aligned}
$$

Then for each $\omega$ subject to (14) there exists a counterexample $f_{\omega} \in R$ such that

$$
\tau_{2}\left(f_{\omega}, \delta\right)=O\left(\omega\left(\delta^{2}\right)\right), \quad \bar{\int} \sup _{k \geqq n}\left|T_{k} f_{\omega}-f_{\omega}\right| \neq c\left(\omega\left(\mu_{n}\right)\right)
$$

Proof: To construct the elements $h_{n}$, following [5], let $H$ be four times continuously differentiable on $\mathbf{R}$ with

$$
0 \leqq H(u) \leqq 1, \quad H(u)=\left\{\begin{array}{l}
u^{2},|\dot{u}| \leqq 1 / 2, \\
0,|u| \geqq 1 .
\end{array}\right.
$$

Choose a constant $c_{5}>0$ such that (cf. (9)) $K_{0}:=2 K c_{5}^{2} \leqq c_{4} c_{5}-\left\|H^{(4)}\right\|_{B} K c_{5}^{2} / 24$ and define

$$
d_{n}=\left(\mu_{n} / c_{5}\right)^{1 / 2}, \quad h_{x n}(u)=H\left((u-x) / d_{n}\right), \quad u, x \in[a, b] .
$$

Without loss of generality let $x_{j n}:=\left(a_{j n}+b_{j n}\right) / 2$ satisfy $\left|x_{j n}-x_{j \pm 1, n}\right| \geqq 3 d_{n}$ (otherwise omit some intervals, namely each time at most $m$ ones, where $m$, independent of $n$, is determined by' $m c_{3} \geqq 3 c_{5}^{-1 / 2}$. Now set

$$
\grave{h}_{n}=\sum_{j=1}^{j_{n}} h_{j n}, \quad h_{j n}=h_{x_{j n}, n} .
$$

Let $u \in[a, b]$ with $h_{j n}(u) \neq 0$. Then $\left|u-x_{j n}\right| \leqq d_{n}$ which implies $\left|u-x_{i n}\right|>d_{n}$, thus $h_{\text {in }}(u)=0$ for $i \neq j$. Therefore one has (15) since $\left\|h_{n}\right\|_{B}=\max _{j}\left\|h_{j n}\right\|_{B}=\|H\|_{B}$ $\leqq 1$. Moreover (cf. [15, p. 24]), one obtains (16) in view of (19) and

$$
\tau_{2}\left(h_{n} ; \delta\right) \leqq C \delta^{2}\left\|h_{n}^{\prime \prime}\right\|_{1} \leqq C \delta^{2} d_{n}^{-1} j_{n} \int_{-1}^{1}\left|H^{\prime \prime}(u)\right| d u \leqq C \delta^{2} / \mu_{n}
$$

Now let. $x, y \in I_{n}$. with $|x-y| \leqq d_{n} / 2$. By (21) one has $H^{\prime \prime}\left((x-y) / d_{n}\right)=2$, $H^{(3)}\left((x-y) / d_{n}\right)=0$ so that for $u \in[a, b]$ there exists $\xi$ between $x$ and $u$ with

$$
\begin{aligned}
h_{y n}(u)= & h_{y n}(x)+(u-x) H^{\prime}\left((x-y) / d_{n}\right) / d_{n}+(u-x)^{2} / d_{n}{ }^{2} \\
& +(u-x)^{4} \cdot H^{(4)}\left((\xi-y) / d_{n}\right) / 24 d_{n}{ }^{4} .
\end{aligned}
$$

In view of $(8),(9),(20)$ this gives

$$
\begin{aligned}
\left|T_{n}\left(h_{y n} ; x\right)-h_{y n}(x)\right|^{\prime} & \geqq T_{n}\left((u-x)^{2} ; x\right) / d_{n}^{2}-T_{n}\left((u-x)^{4} ; x\right)\left\|H^{(4)}\right\|_{B} / 24 d_{n}^{4} \\
& \geqq c_{4} \mu_{n} / d_{n}^{2}-K \mu_{n}^{2}\left\|H^{(4)}\right\|_{B} / 24 d_{n}^{4} \\
& =c_{4} c_{5}-\left\|H^{(4)}\right\|_{B} K c_{5}{ }^{2} / 24 \geqq K_{0},
\end{aligned}
$$


i.e., with. $y=x_{i n}$ one has

$$
\left|T_{n}\left(h_{i n} ; x\right)-h_{j n}(x)\right| \geqq K_{0} \quad\left(x \in I_{n},\left|x-x_{j n}\right|<d_{n} / 2\right) .
$$

Setting

$$
\psi_{n}^{\prime}=\min \left\{d_{n} / 2, c_{3} \mu_{n}^{1 / 2} / 2\right\}=: c_{6} \mu_{n}^{1 / 2}, B_{n}=\bigcup_{j=1}^{j_{n}}\left[x_{i n}-\psi_{n}, x_{j n}+\psi_{n}\right],
$$

$B_{n}$ is of total length $j_{n} 2 \psi_{n} \geqq 2 c_{6} c_{1}>0$ by (19).

Now consider $x \in B_{n}$ which implies $\left|x-x_{k n}\right| \leqq d_{n} / 2$ for some $1 \leqq k \leqq j_{n}$. Since $\left|x-x_{j n}\right|>d_{n}$ for $j \neq k$, one has $h_{j n}(x)=0$, thus in view of (23)

$$
\left|T_{n}\left(h_{n} ; x\right)-h_{n}(x)\right| \geqq \dot{K}_{0}-T_{n}\left(\sum_{j \neq k} h_{j n}(u) ; x\right) \text {. }
$$

If $u \in[a, b]$ satisfies $|x-u| \leqq d_{n}$, then

$$
\left|x_{k \pm 1, n}-u\right| \geqq\left|x_{k \pm 1, n}-x_{k n}\right|_{\mathrm{i}}-\left|x_{k n}-x\right|-|x-u| \geqq d_{n},
$$

thus $h_{j n}(u)=0$ for $j \neq k$ so that $\sum_{j \neq k} h_{j n}(u)=0$ for $|x-u| \leqq d_{n}$. On the other hand, if $|x-u|>d_{n}$, then $\sum_{j \neq k} \dot{h}_{j n}^{\prime}(u) \leqq 1 \leqq\left((x-u) / d_{n}\right)^{4}$. Altogether this. delivers $\sum_{j \neq k} h_{j n}(u) \leqq\left((x-u) / d_{n}\right)^{4}$. for $u \in[a, b]$ which gives in connection with (9), (24)

$$
\left|T_{n}\left(h_{n} ; x\right)-h_{n}(x)\right| \geqq K_{0}-K \mu_{n}^{2} / d_{n}{ }^{4}=K_{0}-K c_{5}{ }^{2}=K_{0} / 2 \quad\left(x \in B_{n}\right) .
$$

This establishes (17) since

$$
R_{n} h_{n} \geqq \int_{B_{n}} \backslash \backslash T_{n} h_{n}-h_{n} \mid \geqq c_{6} \dot{c}_{1} K_{0}>0
$$

For $U_{\delta} f=\tau_{1}(f, \delta)$ one may analogously deduce the following contribution concerning the sharpness of Theorem 1 (cf. (22)).

- Corollary 6: Under the assumptions of Theorem $5^{-}$there exists a counterexample. $f_{\omega} \in R$ such that

$$
\tau_{1}\left(f_{\omega}, \delta\right)=O(\omega(\delta)), \quad \bar{\int} \sup _{k \geq n}\left|T_{k} \dot{f}_{\omega}-f_{\omega}\right| \neq o\left(\omega\left(\mu_{n}{ }^{1 / 2}\right)\right)
$$

Note that the proof still works (cf. (25)) if one considers $R_{n} f:=\int\left|T_{n} f-f\right|$ so that one also obtains the sharpness of estimates of type

$$
\int\left|T_{n} f-f\right| \leqq C \tau_{2}\left(f, \mu_{n}^{1 / 2}\right) \quad(f \in R),
$$

thus even for the $L^{1}$-error (cf. (10)).

\section{Applications}

Let us discuss the preceding Bohman-Korovkin theorem and its sharpness in connection with Bernstein polynomials and spline approximation.

- The. Bernstein polynomials are defined for $f \in R[0,1]$ by

$$
B_{n}(f ; x)=\sum_{k=0}^{n} f\left(\frac{k}{n}\right)\left(\begin{array}{l}
n \\
k
\end{array}\right) x^{k}(1-x)^{n-k}
$$

One has $B_{n} p_{\mathrm{i}}=p_{\mathrm{i}}$ for $i=0 ; 1$ and $B_{n}\left((u-x)^{2} ; x\right)=\varphi(x) / n, \varphi(x):=x(1-x)$, thus (8), (19), (20) with $\mu_{n}=1 / 4 n$ are fulfilled if one chooses

$$
I_{j n}=\left(1 / 4+(j-1) n^{-1 / 2}, 1 / 4+j n^{-1 / 2}\right), \quad n^{1 / 2} / 2 \leqq j_{n}<n^{1 / 2} / 2+1 .
$$


Concerning $(9)$ only note that $B_{n}\left((u-x)^{4} ; x\right)=3 \varphi^{2}(x) / n^{2}+\varphi(x)(1-6 \varphi(x)) / n^{3}$.' Hence our theorems deliver

Corollary $7:$ For $f \in R[0,1]$ there holds true

$$
-\bar{\int} \sup _{k \geq n}\left|B_{k} f-f\right| \leqq C \tau_{2}\left(f, n^{-1 / 2}\right) \text {. }
$$

Moreover, for each $\omega$ subject to (14) there exists an $f_{\omega} \in R[0,1]$ such that

$$
\tau_{2}\left(f_{\omega}, \delta\right)=O\left(\omega\left(\delta^{2}\right)\right), \quad \bar{\int} \sup _{k \geqq n}\left|B_{k} f_{\omega}-f_{\omega}\right| \neq o(\omega(1 / n)) .
$$

Concerning the $L^{1}$-error $\int\left|B_{n} f-f\right|$, results like (27) (cf. (26)) are well-known (cf. [1], [15, p. 107]). In fact, instead of (9), convexity arguments are available for $B_{n}{ }^{\prime \prime} g_{\delta}-g_{\delta}^{\prime \prime}$ (cf. (12)). Though (27) is sharp in the sense of (28), no inverse theorem can be established. Therefore, in [10] the relevant $L^{\hat{1}}$-estimate was strengthened by substituting the (unweighted) $\tau_{2}$-modul by

$$
\tau_{2}{ }^{\varphi}(f, \delta):=\bar{\int} \sup \left\{\left|\Delta_{h}^{2} f(t)\right|: t \pm h \in U_{\delta \varphi^{1 / 2}(x)+\delta^{2}}(x)\right\} d x .
$$

Partial inverse theorems were then established in [11] for the $L^{p}$-error, $p>1$. Concerning the Riemann error $\bar{\int} \sup _{k \geqq n}\left|B_{k} f-f\right|$ discușsed here, an improved direct as well as inverse result can be found in [18], namely

$$
\left\|\sup _{k \geqq n}\left|B_{k} f-f\right|\right\|_{1 / n}^{\Phi} \leq M \tau_{2}{ }^{\varphi}\left(f, n^{-1 / 2}\right), \quad \tau_{2}{ }^{\varphi}\left(f, n^{-1 / 2}\right) \leqq \frac{M}{n} \sum_{k=1}^{n}\left\|\sup _{m \geq k}\left|B_{m} f-f\right|\right\|_{1 / k}^{\rho}
$$

in terms of the weighted norm

$$
\cdot\|f\|_{\delta^{2}}^{\varphi}=\int \sup \left\{|f(y)|: y \in U_{\delta \phi^{i / 2}(x)+\delta^{2}}(x)\right\} d x .
$$

Iet us also mention that the sharpness of the latter results is discussed in [17].

Finally consider linear interpolating splines on the equidistant nodes $x_{k n}:=k / n$ in the interval $[0,1]$. With the continuous functions

$$
g_{k n}(x)= \begin{cases}1-n\left|x_{k n}-x\right|, & \left|x_{k n}-x\right| \leqq 1 / n, \\ 0 & \text { else }\end{cases}
$$

the spline operator $S_{n}^{\prime}$ is defined for $f^{\prime} \in R[0,1]$ by

$$
S_{n}(f ; x)=\sum_{k=0}^{n} f\left(x_{k n}\right) \dot{y}_{k n}(x) \quad(\dot{x} \in[0,1]) .
$$

Obviously, $S_{n} p_{i}=p_{i}$ for $i=0,1$, thus (8). Furthermore, one has $(x \in[0,1], r=2,4)$

$$
S_{n}\left((u-x)^{r} ; x\right)=\sum_{k=0}^{n}\left(x_{k n}-x\right)^{r} g_{k n}(x)=\sum_{g_{k n}(x) \neq 0}\left(x_{k n}-x\right)^{r} g_{k n}(x) \leqq \frac{1}{n^{r}},
$$

since $\left|x_{k n}-x\right| \leqq 1 / n$ if $g_{k n}(x) \neq 0$. Set $I_{n}=\bigcup_{k=1}^{n}((4 k-3) / 4 n,(4 k-1) / 4 n)$. Then $x \in I_{n}$ implies $\left|x_{k n}-x\right| \geqq 1 / 4 n$ which gives

$$
S_{n}\left((u-x)^{2} ; x\right)=\sum_{k=0}^{n}\left(x_{k n}-x\right)^{2} g_{k n}(x) \geqq \frac{1}{16 n^{2}} \sum_{k=0}^{n} g_{k n}(x)=\frac{1}{16 n^{2}}
$$


Therefore $1 / 16 n^{2} \leqq \mu_{n} \leqq 1 / n^{2}$ in view of (29), and (9), (19), (20) with $j_{n}=n$ follow by $(29),(30)$.

Corollary 8: For $f \in R[0,1]$ there holds true

$$
\bar{\int} \sup _{k \geqq n}\left|S_{k} f-f\right| \leqq C \tau_{2}(f, 1 / n)
$$

and for each $\omega$ subject to (14) there exists an $f_{\omega} \in R[0,1]$ such that

$$
\ddot{\tau_{2}}\left(f_{\omega}, \delta\right)=O\left(\omega\left(\delta^{2}\right)\right), \quad \bar{\int} \sup _{k \geq n}\left|S_{k} f_{\omega}-f_{\omega}\right| \neq c\left(\omega\left(1 / n^{2}\right)\right)
$$

The result, corresponding to (31), for the $L^{1}$-error; is again well-known (cf. [15, p: 123]).

\section{REFERENCES}

[1] Andreev, A. S., and V. A. Popov: Approximation of functions by means of linear summation operators in $L_{p}$. In: Functions, Series, Operators I. Proc. Conf. Budapest 1980 (Eds.: B. Sz.-Nagy and J. Szabados). Amsterdam: North-Holland 1983, pp. 127-150.

[2] Брудныи, Ю. А.: Приближение функций $n$ перемениых квазимного-членами. Изв. Акад. Наук СССР, Сер. мат. $34(1970), 564-583$.

[3] DeVore, R. A.: The Approximation of Continuous Functions by Positive Linear Operators (Lecture Notes in Mathematics 293). Berlin: Springer-Verlag 1972.

[4] DeVore, R. A.: Inverse theorems for approximation by positive linear operators. In: Spline Functions and Approximation Theory. Proc. Conf. Edmonton 1972 (Eds.: A. Meir and A: Sharma). Basel: Birkhäuser Verlag 1973, pp. 371-375.

[5] Dickmers, $W_{::}$On quantitative condensation of singularities on sets of full measure (to appear).

[6] Dickmeis, W., Mevissen, H., Nessel, R. J., and E. van Wickeres: Sequential convergence and approximation in the space of Riemann integrable functions. J. Approx. Theory (in print).

[7] Dickmeis, W., Nessel, R. J., and E. van Wickeren: Quantitative extensions of the uniform boundedness principle. Jahresber. Deutsch. Math.-Verein. 89 (1987), 105-134.

[8] Freud, G.: On approximation by positive linear methods, II. Studia Sci. Math. Hungar. 3 (1968), 365-370.

[9] Gonska, H. H.: Quantitative Korovkin type theorems on simultaneous approximation. Math. Z. 186 (1984), $419-433$.

[10] Ivanov, K. G.: Approximation by Bernstein polynomials in $L_{p}$ metric. In: Constructive Theory of Functions. Proc. Conf. Varna 1984 (Eds.: BI. Sendov et al.). Sofia: Publ. House Bulg. Acad. Sci. 1984, pp. 421-429.

[11] Ivanov, K. G.: Converse theorems for approximation by Bernstein polynomials in $L_{p}[0,1]$ $(1<p<\infty)$. Constr. Approx. 2 (1986), 377-392.

[12] Mevissen, H., Nessel, R. J., and E. van' Wickeren: On the Ricmann convergence of positive linear operators. In: Proc. Constructive Function Theory '86, Edmonton. Rocky Mountain J. Math. (in print).

[13] Popov, V. A.: Average moduli and their function spaces. In : Constructive Function Theory '81. Proc. Conf. Varna 1981 (Eds.: Bl. Sendov et al.). Sofia: Publ. House Bulg. Acad. Sci. 1983 , pp. $482-487$.

[14] QరАK, E.: Uni- und multivariate $L_{p}$-Abschätzungen des Approximationsfehlers positiver linearer Operatoren mit Hilfe des $\tau$-Moduls. Dissertation, Universität Dortmund 1985.

[15] Sendov, Bl., and V. A. Popov: Averaged Moduli of.Smoothness (Bulgarian). Sofia: Publ: House Bulg. Acad. Sci. 1983. 
[16] Trmax, A. F.: Theory of Approximation of Functions of a Real Variable. New York: Pergamon Press 1963.

- [17] vá Wickeren, E.: On the approximation of Riemann intègrable functions by Bernstein polynomials (to appear).

[18] VAN WICKEREN, E.: Direct and inverse, theorems for Bernstein polynomials in the space of Riemann integrable functions (to appear).

Manuskripteingang: 11.08. 1987

VERFASSER:

H. Mevissen, R. J. Nessel und E. van Wickeret Lehrstuhl A für Mathematik der Rheinisch-Westfälischen Technischen Hochschule Templergraben 55 D. 5100 Aachen. 